\title{
Prostatectomía radical versus crioablación: supervivencia de los pacientes
}

Ramírez-Buensuceso-Conde NA, ${ }^{1}$ Ávalos-Jiménez JC, ${ }^{2}$ Xochipiltecatl-Muñoz $\mathrm{JD},{ }^{3}$ Cruz-Zárate A, ${ }^{4}$ Robles-Aviña JA, ${ }^{5}$ Luna-Martínez J6

\section{Resumen}

ANTECEDENTES: el cáncer de próstata es el tumor maligno más frecuente en varones mayores de 50 años edad. La crioablación es una técnica que provoca menos complicaciones y efectos adversos, y mayor calidad de vida que la prostatectomía. Aunque sus ventajas son bien conocidas, por el momento no existen estudios relacionados con la supervivencia después del procedimiento.

OBJETIVO: comparar la supervivencia de pacientes con cáncer de próstata intervenidos mediante prostatectomía radical versus crioablación.

MATERIALES Y MÉTODOS: estudio retrospectivo, observacional y descriptivo, en el que se utilizó el Sistema Integral de Administración Hospitalaria de PEMEX para obtener los datos de los expedientes electrónicos en la pestaña de "Reportes". Se seleccionaron los pacientes con diagnóstico de cáncer de próstata, con reporte histopatológico completo, intervenidos mediante prostatectomía o crioablación, atendidos en el Hospital Central Sur de Alta Especialidad de PEMEX entre enero de 2005 y enero de 2016. Se utilizó la prueba de $\chi^{2}$ para el análisis de mortalidad de grupos independientes. Se consideró estadísticamente significativo el valor de $p<0.05$

RESULTADOS: se registraron 289 expedientes. El promedio de edad fue de 62 años. Se reportaron 7 (2.4\%) defunciones en el grupo de prostatectomía radical y $6(2.1 \%)$ en el de crioablación $(p=0.41)$. De los pacientes fallecidos, 7 resultaron con concentración de antígeno prostático específico mayor de $1 \mathrm{ng} / \mathrm{mL}$ y 6 con concentración menor de $1 \mathrm{ng} / \mathrm{mL}$.

CONCLUSIONES: no existe diferencia estadísticamente significativa en la supervivencia de los pacientes intervenidos mediante prostatectomía radical versus crioablación. Se requieren estudios adicionales que evalúen los efectos adversos, costos de atención y control de la enfermedad a largo plazo para considerar que la crioablación es superior a la prostatectomía radical.

PALABRAS CLAVE: supervivencia a la crioablación; prostatectomía radical; cáncer de próstata.

\footnotetext{
${ }^{1}$ Residente de cuarto año, Departamento de Cirugía General, Hospital Central Sur de Alta Especialidad de PEMEX, Ciudad de México.

2 Residente de primer año, Departamento de Urología, Hospital Regional Licenciado Adolfo López Mateos, Ciudad de México.

${ }^{3}$ Jefe de servicio, Departamento de Urología.

${ }^{4}$ Titular del curso, Departamento de Cirugía General.

${ }^{5}$ Jefe de servicio, Departamento de Oncología.

${ }^{6}$ Jefe de servicio, Departamento de Cirugía General. Hospital Central Sur de Alta Especialidad de PEMEX, Ciudad de México.
}

Recibido: diciembre 2016

Aceptado: octubre 2017

Correspondencia

Nubia Andrea Ramírez Buensuceso Conde nubiandrea@gmail.com

Este artículo debe citarse como

Ramírez-Buensuceso-Conde NA, Ávalos-Jiménez JC, Xochipiltecatl-Muñoz JD, Cruz-Zárate A, Robles-Aviña JA, Luna-Martínez J. Prostatectomía radical versus crioablación: supervivencia de los pacientes. Rev Mex Urol. 2017 nov-dic;77(6):437-445.

DOI: https://doi.org/10.24245/revmexurol.v77i6.1127 
Rev Mex Urol. 2017 Nov-Dec;77(6):437-445.

\section{Radical prostatectomy versus cryoablation: Survival of patients}

\author{
Ramírez-Buensuceso-Conde NA, ${ }^{1}$ Ávalos-Jiménez JC, ${ }^{2}$ Xochipiltecatl-Muñoz \\ JD, ${ }^{3}$ Cruz-Zárate A, ${ }^{4}$ Robles-Aviña JA, ${ }^{5}$ Luna-Martínez J ${ }^{6}$
}

\begin{abstract}
BACKGROUND: Prostate cancer is the most frequent malignant tumor in men above 50 years of age. Cryoablation has fewer complications and adverse effects, as well as offering better quality of life than prostatectomy. Even though its advantages are well-known, there are currently no studies related to survival after the procedure.
\end{abstract}

OBJECTIVE: To compare survival in patients that underwent radical prostatectomy versus cryoablation.

MATERIALS AND METHODS: A retrospective, observational, and descriptive study was conducted that utilized the Integrated Hospital Administration System of PEMEX to review electronic case records, utilizing the "Reports" tab. Patients diagnosed with prostate cancer that had complete histopathologic studies and that underwent prostatectomy or cryoablation at the Hospital Central Sur de Alta Especialidad de PEMEX within the time frame of January 2005 and January 2016 were selected. The mortality analysis for independent groups was carried out using the $\chi^{2}$ test and statistical significance was set at a $p<0.05$.

RESULTS: A total of 289 patient files were obtained and the mean age of the patients was 62 years. Seven (2.4\%) patients in the radical prostatectomy group died and $6(2.1 \%)$ patients in the cryoablation group died $(p=0.41)$. Of the patients that died, 7 had a prostate-specific antigen level above $1 \mathrm{ng} / \mathrm{mL}$ and 6 had a prostate-specific antigen level below $1 \mathrm{ng} / \mathrm{mL}$.

CONCLUSIONS: There was no statistically significant difference in survival between patients that underwent radical prostatectomy versus cryoablation. Further studies that evaluate adverse effects, costs, and long-term disease control are needed to determine whether cryoablation is superior to radical prostatectomy.

KEYWORDS Survival in cryoablation; Radical prostatectomy; Prostate cancer

\footnotetext{
${ }^{1}$ Residente de cuarto año, Departamento de Cirugía General, Hospital Central Sur de Alta Especialidad de PEMEX, Ciudad de México.

${ }^{2}$ Residente de primer año, Departamento de Urología, Hospital Regional Licenciado Adolfo López Mateos, Ciudad de México.

3 Jefe de servicio, Departamento de Urología.

${ }^{4}$ Titular del curso, Departamento de Cirugía General.

${ }^{5}$ Jefe de servicio, Departamento de Oncología.

${ }^{6}$ Jefe de servicio, Departamento de Cirugía General.

Hospital Central Sur de Alta Especialidad de PEMEX, Ciudad de México.
}

Correspondence

Nubia Andrea Ramírez Buensuceso Conde nubiandrea@gmail.com

\section{ANTECEDENTES}

El cáncer de próstata es el tumor maligno más frecuente en varones de 50 años de edad, in- cluso ha superado al cáncer de pulmón en este grupo de pacientes. Este cambio se asoció con el desarrollo del antígeno prostático específico (APE), que permitió detectar mayor número de 
casos de cáncer de próstata en estadios tempranos. Los índices de mortalidad por cáncer de próstata ajustados por edad disminuyeron 4.1\% anualmente de 1994 a 2001; sin embargo, en 2014 se registraron 29,480 defunciones por esta neoplasia. Este índice de mortalidad, comparativamente bajo, hace necesaria (salvo que el cáncer de próstata se esté volviendo biológicamente menos agresivo) una mayor conciencia para la detección temprana y tratamiento oportuno del tumor, antes de elevarse su incidencia. No obstante, cuando no supone una amenaza a la expectativa de vida (supervivencia), provoca efectos secundarios que afectan la calidad de vida y aumentan los gastos de atención médica. ${ }^{1}$

El Instituto Nacional del Cáncer ( $\mathrm{NCl}$ ) informa que la tasa relativa de supervivencia a 5 años es de $100 \%$, a 10 de $98 \%$ y a 15 de $95 \%$ del cáncer de próstata. Esta tasa disminuye al dividirse por estadios, en el que la metástasis a distancia es la única etapa con diferente índice de supervivencia $(28 \%){ }^{2}$

En México, el cáncer de próstata es la neoplasia con mayor índice de mortalidad en hombres, con una tasa de 13 por cada 100,000 habitantes. En 2008 se registró un promedio de vida de 75 años en la población mexicana. Por lo anterior, la incidencia, morbilidad y mortalidad por cáncer de próstata se encuentra en aumento. Un estudio realizado en el Instituto Nacional de Salud Pública reportó una mortalidad por cáncer de próstata de 3.9 por cada 10,000 habitantes entre 1980 y 1984 , de 5.2 entre 1995 y 1999, y de 5.5 en 2005 a 2009; esto supone un aumento sostenido de aproximadamente $2 \%$ anual. ${ }^{3}$

Aunque su origen es desconocido, se han descrito factores de riesgo como: edad mayor de 50 años, ascendencia africana, antecedentes familiares de la neoplasia, consumo de carnes rojas y lácteos, tabaquismo, obesidad y concentraciones elevadas de testosterona. ${ }^{4}$ Un metanálisis reportó que el tabaquismo aumenta el riesgo de mortalidad por cáncer de próstata (riesgo relativo de 1.24; IC95\%: 1.18-1.31), ${ }^{5}$ incluso se ha señalado que a mayor edad aumenta la posibilidad de obtener resultados falsos positivos. ${ }^{6}$

Los síntomas suelen ser inespecíficos. Al inicio de la enfermedad no se manifiestan síntomas y después aparece prostatismo en estadios avanzados o síntomas asociados con metástasis ósea; por tanto, no deben tomarse en cuenta para establecer el diagnóstico, debido a que los pacientes con enfermedad benigna, como hiperplasia prostática, suelen tener el mismo curso. ${ }^{1}$

En 1980 se introdujo el antígeno prostático específico (APE) como método de tamizaje. Éste ha permitido la identificación temprana, estimando un riesgo de neoplasia maligna o enfermedad metastásica, así como un seguimiento y detección de enfermedad recurrente, por lo que se ha logrado detectar el tumor en etapas más tempranas, contando con mayor incidencia (casos nuevos registrados). ${ }^{2,7}$

El antígeno prostático específico es una glucoproteína sintetizada por las células epiteliales de la próstata, es decir, se trata de un marcador órgano-específico, no así de un marcador de malignidad. Diversos estudios señalan que puede encontrarse elevado en pacientes con hipertrofia prostática benigna, prostatitis, etc.

Aunque la concentración del antígeno prostático no representa un criterio diagnóstico, orienta a la toma de decisiones en pacientes con alta sospecha de malignidad. Pueden realizarse determinaciones adicionales como: fracción libre, densidad y velocidad de aumento o disminución del antígeno. El tacto rectal anormal con concentración elevada (4-10 ng/mL) sugiere sospecha de la enfermedad. Específicamente, el valor mayor de $10 \mathrm{ng} / \mathrm{mL}$ es indicativo de biopsia transrectal. ${ }^{8}$ Existen factores adicionales que de- 
ben considerarse para determinar si un paciente es ideal para biopsia.

Algunos estudios indican que el tamizaje del antígeno prostático específico es efectivo en pacientes de 70 años de edad. Otras investigaciones señalan que a mayor edad, mayor puntaje de la escala de Gleason. Este hallazgo, aunado al aumento de esperanza de vida en los últimos años, debe considerarse para establecer el tratamiento. El puntaje de la escala de Gleason es la suma de ambos valores asignados. El grado de diferenciación suele asociarse con el pronóstico de la enfermedad, por ejemplo: Grado 1 (2-4): bien diferenciado, Grado 2 (5-6): moderadamente diferenciado y Grado 3 (7-10): pobremente diferenciado. Por tanto, se ha sugerido que la escala de Gleason es uno de los factores más importantes en el pronóstico.

Un estudio efectuado en 2015 reportó puntajes de la escala de Gleason de 8 a 10 en hombres de $50-54$ (8.9\%), $70-74$ (16.2\%) y 80-84 (28.5\%) años de edad, clasificándolos en alto grado a 14.3, 22.4 y $38.7 \%$, respectivamente, $(p<0.001) .{ }^{9}$

En conjunto con la determinación del antígeno prostático específico, el grado histológico y la escala de Gleason permiten establecer la estadificación del riesgo y, de esta manera, implementar el tratamiento específico, con mejor pronóstico para el paciente. Esta clasificación fue descrita por D'Amico en 1998. ${ }^{10}$

Los pacientes con riesgo bajo son ideales para vigilancia, con obtención de biopsias y prostatectomía radical, radioterapia externa o braquiterapia. Los sujetos con riesgo intermedio tienen las mismas opciones y los de alto riesgo requieren terapia hormonal con prostatectomía radical, radioterapia, o solo monoterapia.

Otros factores a tomar en cuenta son la esperanza de vida o supervivencia al momento del diagnós- tico, así como enfermedad metastásica ósea o pélvica, afección nodular, entre otras comorbilidades que pueden evaluarse en estudios de extensión, como tomografía y resonancia magnética.

La National Comprehensive Cancer Network ${ }^{11}$ considera cinco grupos de riesgo: 1) muy bajo, 2) bajo, 3) alto, 4) muy alto y 5) metastásico. Esta clasificación supone "enfermedad clínicamente localizada" a los estadios: muy bajo, bajo, intermedio y alto; "enfermedad localmente avanzada" al estadio muy alto y "enfermedad metastásica" a la metástasis. A partir de esta clasificación se considera la supervivencia esperada a 10 o 20 años para iniciar el tratamiento quirúrgico, radioterapia o tratamiento hormonal. ${ }^{11}$

Los criterios de supervivencia más utilizados son el estadio clínico T1c, APE $<0.15 \mathrm{ng} / \mathrm{mL}$, Gleason $\leq 6$, biopsias positivas $\leq 2$ y detección de cáncer en cualquier muestra $\leq 50 \% .^{12}$

La prostatectomía radical es el tratamiento de elección en pacientes cáncer de próstata localizado, principalmente en los de riesgo bajo e intermedio, con esperanza de vida mayor de 10 años, y tiene una supervivencia a 10 años arriba de $94 \%$.

Debido a la alta tasa de márgenes quirúrgicos positivos (33-66\%), entre 56 y $78 \%$ requiere tratamiento coadyuvante con radioterapia o terapia hormonal. La prostatectomía no se recomienda en pacientes con enfermedad localmente avanzada; sin embargo, se ha visto que acompañada de linfadenectomía de la fosa obturatriz y de los vasos iliacos internos y externos puede tener mejor pronóstico, pero mayor morbilidad asociada. ${ }^{1}$ Con la prostatectomía radical, la expectativa de supervivencia libre de enfermedad varía de 80$88 \%$ a 5 años y de $69-75 \%$ a 10 años. ${ }^{12}$

En 1996 la crioablación se reconoció como opción de tratamiento para el cáncer de prós- 
tata por la Asociación Americana de Urología. Esta técnica consiste en la congelación in situ y controlada del tejido, con la finalidad de erradicar la enfermedad y conservar la integridad anatómica. $^{13}$

Las indicaciones actuales para la criocirugía en pacientes con cáncer de próstata incluyen: tumor localizado, fracaso bioquímico posterior a la prostatectomía o radioterapia y control de las complicaciones locales en sujetos con enfermedad diseminada. ${ }^{14}$

La supresión androgénica es el tratamiento paliativo de la enfermedad diseminada. Suelen prescribirse análogos de la hormona liberadora de hormona luteinizante (LHRH); sin embargo, se han reportado múltiples reacciones adversas como: pérdida de la libido, disfunción eréctil, pérdida de masa ósea, sarcopenia, alteraciones lipídicas, resistencia a la insulina y síndrome metabólico.

Diversos estudios detallan el lineamiento a seguir, según el riesgo establecido del paciente. Las generalidades se han expuesto previamente; sin embargo, no es concluyente el algoritmo de tratamiento, quizá debido a la heterogeneidad de los estudios. Aunque existen guías clínicas y algoritmos preestablecidos, el protocolo de tratamiento sigue siendo amplio, lo que conlleva a diferentes pronósticos. Aún se discute acerca de los efectos adversos, pues existe poca diferencia en la supervivencia a 10 años.

Un estudio realizado en 2006, en el Walter Reed National Military Medical Center en Bethesda, Maryland, en el que se evaluó a pacientes con riesgo bajo e intermedio que escogieron su tratamiento, reportó que $54 \%$ de los casos fueron tratados con cirugía, 20\% con radiación externa y $12 \%$ con vigilancia, mientras que en los pacientes con alto riesgo: $34 \%$ prefirieron cirugía y $57 \%$ radiación externa con tratamiento hor- monal. La elección del tratamiento fue diferente según la edad al momento del diagnóstico, raza y comorbilidades. La mayoría de los pacientes se mostraron satisfechos con su decisión de tratamiento. ${ }^{15}$

Los pacientes con cáncer de próstata cuentan con diversas opciones de tratamiento, pues casi todas reportan similar eficacia, pero diferentes efectos adversos. Actualmente se discute el uso de escalas y la toma de decisiones; por tanto, cada tratamiento debe individualizarse.

La crioablación provoca menos complicaciones y efectos adversos, y mayor calidad de vida que la prostatectomía. Generalmente, el promedio de días de hospitalización es de 1 a 2 días. Las complicaciones más frecuentes se asocian con retención y estenosis urinaria, e infección de vías urinarias en 17,5 y $17 \%$, respectivamente.

La función urinaria se preserva en 95-100\% de los casos, mientras que la función eréctil en 54$100 \%$. La toxicidad rectal se ha reportado muy poco. La tasa de fístula rectouretral es de $1 \%$.

El control bioquímico a 8 años de seguimiento es de $86.2 \%$. La evolución a metástasis no está reportada en la mayor parte de los estudios, pero se estima tan baja como $0.3 \%$, aproximadamente. ${ }^{16-18}$

En el Hospital Central Sur de Alta Especialidad de PEMEX se implementa la crioablación para el tratamiento de pacientes con cáncer de próstata localizado (órgano confinado) desde 2005. La prostatectomía radical se realiza, de igual forma, en sujetos con riesgo bajo e intermedio, principalmente. El tiempo quirúrgico reportado con ambos procedimientos, en nuestra institución y en el Hospital Licenciado Adolfo López Mateos (ISSSTE), es de 3 a 5 horas. El sangrado estimado con crioterapia y prostatectomía es de 30-100 y $800-12,500 \mathrm{~mL}$, respectivamente. En relación 
con la estancia hospitalaria, los pacientes con crioterapia permanecen entre 3 y 7 días, y con prostatectomía radical de 4 a 8 días. ${ }^{13,19}$

Aunque las ventajas transoperatorias y posoperatorias son bien conocidas, el tratamiento de elección sigue siendo la prostatectomía radical. El beneficio en cuanto a supervivencia no se ha evaluado en México, lo que supone una ventaja más para la crioablación.

El objetivo de este estudio es comparar la supervivencia en pacientes con diagnóstico de cáncer de próstata con prostatectomía radical versus crioablación en el Hospital Central Sur de Alta Especialidad de PEMEX.

\section{MATERIALES Y MÉTODOS}

Estudio retrospectivo, observacional y descriptivo, que utilizó el Sistema Integral de Administración Hospitalaria de PEMEX para obtener los datos de los expedientes electrónicos en la pestaña de "Reportes". Se seleccionaron pacientes con diagnóstico de cáncer de próstata, con reporte histopatológico completo, con prostatectomía o crioablación, atendidos en el Hospital Central Sur de Alta Especialidad de PEMEX entre enero de 2005 y enero de 2016. Posteriormente se evaluaron las variables: determinación del antígeno prostático específico (APE) pre y posquirúrgico, clasificación de la escala de Gleason, tipo de procedimiento (prostatectomía o crioablación), edad al momento del diagnóstico, edad actual en caso supervivencia y causa de muerte en caso de fallecimiento. Se elaboró una base de datos por año para proceder al análisis estadístico.

Los datos se analizaron con estadística analítica, con medias y desviación estándar para variables cuantitativas, y frecuencias y porcentajes para variables cualitativas. Se utilizó la prueba de $\chi^{2}$ para el análisis de mortalidad de grupos independientes. Para todas las pruebas se utilizó el programa SPSS v15; se consideró estadísticamente significativo el valor de $p<0.05$.

\section{RESULTADOS}

Se registraron 289 expedientes de pacientes con cáncer de próstata. El promedio de edad fue de 62 años (límites mínimo y máximo de 44 y 83 años, respectivamente); concentración de antígeno prostático específico prequirúrgico de 13.1 $\mathrm{ng} / \mathrm{mL}$ y posquirúrgico de $2.63 \mathrm{ng} / \mathrm{mL}$.

Al dividir los grupos en prostatectomía radical y crioablación (Cuadro $\mathbf{1}$ ) se encontró un promedio de edad al momento del diagnóstico similar en ambos grupos (61 y 64 , respectivamente), al igual que en la concentración de antígeno prostático específico prequirúrgico: $13.4 \pm 36.7$ en el grupo de prostatectomía y $11.1 \pm 9.6$ en el grupo de crioablación. Al analizar el APE posquirúrgico se observó $2.5 \pm$ 20.2 y $2.7 \pm 10$, respectivamente (Figura 1). La escala de Gleason resultó con $6.5 \pm 1.6$ para el grupo de prostatectomía y con $6.1 \pm 1.8$ para crioablación.

En lo que a mortalidad respecta, se registraron $7(2.4 \%)$ defunciones en el grupo de prostatectomía radical y $6(2.1 \%)$ en el de crioablación $(p=0.41)$, sin significación estadística. De

Cuadro 1. Características de los pacientes intervenidos mediante prostatectomía o crioablación.

\begin{tabular}{|l|c|c|}
\hline Variable & $\begin{array}{c}\text { Prostatectomía } \\
\text { radical }(\mathbf{n}=\mathbf{1 8 6})\end{array}$ & $\begin{array}{c}\text { Crioablación } \\
(\mathbf{n}=\mathbf{1 0 3})\end{array}$ \\
\hline Edad & $61 \pm 7.1$ & $64 \pm 7.8$ \\
\hline APE inicial & $13.4 \pm 36.7$ & $11.1 \pm 9.6$ \\
\hline Gleason & $6.5 \pm 1.6$ & $6.1 \pm 1.8$ \\
\hline APE final & $2.5 \pm 20.7$ & $2.7 \pm 10$ \\
\hline Mortalidad & $7(2.4 \%)$ & $6(2.1 \%)$
\end{tabular}

APE: antígeno prostático específico; mortalidad $(\mathrm{p}=0.41)$. 


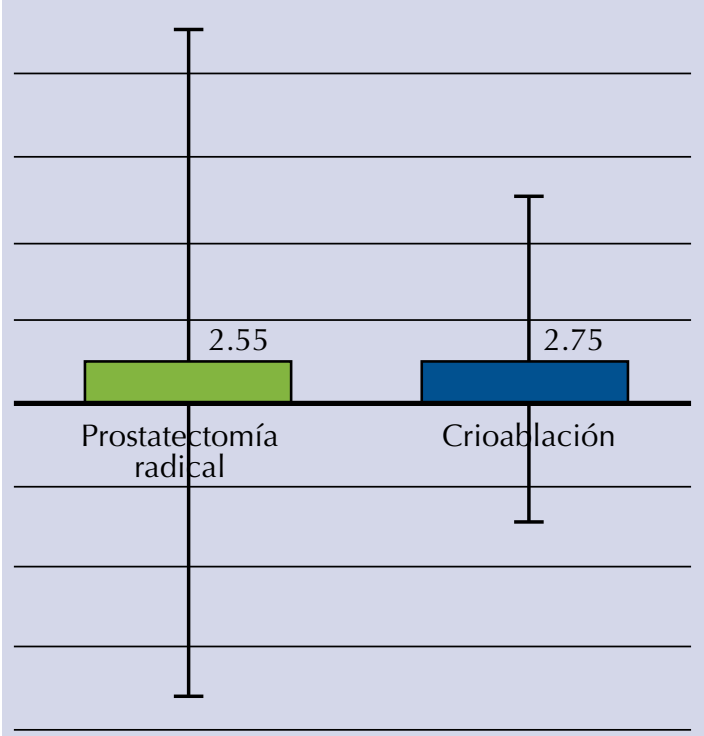

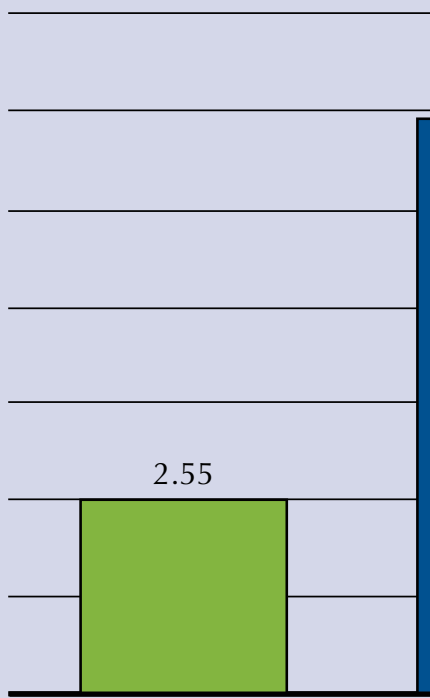

Prostatectomía radical

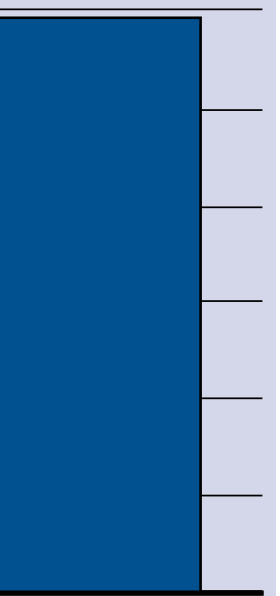

Crioablación

Figura 1. Concentración de antígeno prostático específico en pacientes con prostatectomía radical y crioablación.

todos los pacientes fallecidos, 7 resultaron con concentración de antígeno prostático específico mayor de $1 \mathrm{ng} / \mathrm{mL}$ y 6 con concentración menor de $1 \mathrm{ng} / \mathrm{mL}$.

\section{DISCUSIÓN}

El cáncer de próstata es la neoplasia más frecuente en varones. Actualmente existen múltiples tratamientos enfocados a erradicar o controlar este tumor. Los pacientes con cáncer de próstata localizado son ideales para intervención quirúrgica con fines curativos y, según la evolución durante el seguimiento, terapia coadyuvante. ${ }^{11}$ El tratamiento de elección es la prostatectomía radical.

La crioablación se inició en 1963, pero hasta 1996 la reconoció la Asociación Americana de Urología como una opción efectiva para el cáncer de próstata. ${ }^{13,19}$ En México existen pocos centros donde se practica esta técnica (el Hospital Central Sur de Alta Especialidad PEMEX es uno de ellos). En 2005 se realizaron los primeros procedimientos de crioablación y en 2007 se implementó formalmente como tratamiento en pacientes con cáncer de próstata localizado (órgano confinado). ${ }^{19}$ Este estudio reportó un promedio de edad de 62 años en pacientes tratados con crioablación.

La concentración de antígeno prostático específico posterior al tratamiento fue mínima $(2.5 \pm 20.27$ y $2.75 \pm 10$ en el grupo de prostatectomía radical y crioablación, respectivamente), resultado asociado con mal control de la enfermedad, que aunque se registraron pocos casos, modificó el valor de la desviación estándar.

Este estudio no consideró la terapia coadyuvante ni la concentración de antígeno prostático espe- 
cífico posterior a ésta; por tanto, el resultado es únicamente el reflejo de la enfermedad después del tratamiento quirúrgico, con la determinación del antígeno prostático en la consulta inmediata a la intervención.

El objetivo de este estudio fue comparar la supervivencia de pacientes con prostatectomía radical versus crioablación. Entre los resultados se registraron 7 (2.4\%) defunciones en el grupo de prostatectomía y $6(2.1 \%)$ en el de crioablación, que resultó estadísticamente significativo $(p=0.41)$.

Conocer si los pacientes recibieron tratamiento coadyuvante podría ayudar a determinar la baja mortalidad durante el estudio. Los múltiples tratamientos para el cáncer de próstata han ayudado a mantener un adecuado control de la enfermedad, por lo que el Instituto Nacional del Cáncer $(\mathrm{NCl})$ reporta que la tasa relativa de supervivencia a 5 años es de $100 \%$, a 10 años de $98 \%$ y a 15 de $95 \%$. $^{3}$ Por lo anterior, se requieren estudios prospectivos y con seguimiento a 15 años para determinar la supervivencia de los pacientes con cáncer de próstata.

Las limitantes de este estudio fueron: desconocer los tratamientos coadyuvantes y la causa específica de mortalidad. Se requieren estudios adicionales, a largo plazo, con otro tipo de variables para determinar la supervivencia en este grupo de pacientes.

No se encontró diferencia estadísticamente significativa en la supervivencia de pacientes intervenidos de prostatectomía o crioablación; este dato puede deberse a variables no estudiadas como: tratamiento coadyuvante, comorbilidades o falta de seguimiento; por tanto, la crioablación parece comportarse de forma similar a la prostatectomía en la supervivencia de pacientes con cáncer de próstata.

\section{CONCLUSIONES}

El cáncer de próstata es una neoplasia con elevada incidencia. En el Hospital Central Sur de Alta Especialidad de PEMEX se efectúa la crioablación en pacientes con cáncer de próstata localizado (órgano confinado). No existe diferencia estadísticamente significativa en la supervivencia de pacientes con prostatectomía radical o crioablación. Ambos tratamientos representan una opción efectiva en pacientes con cáncer de próstata en etapa inicial. Aunque las ventajas de la crioablación son ampliamente conocidas, se requieren estudios adicionales, en los que se evalúen los efectos adversos, costos y control de la enfermedad a largo plazo para considerarla superior a la prostatectomía radical.

\section{Fuente de financiamiento}

No se requirió ningún tipo de financiamiento para realizar este trabajo.

\section{Conflictos de interés}

Los autores declaran no tener conflictos de interés.

\section{REFERENCIAS}

1. Morote J, Maldonado X, Morales R, Cáncer de próstata. Med Clin. 2016;146;121-127.

2. American Cancer Society. [en línea]. Dirección URL: <http:// www.cancer.org/cancer/prostatecancer/detailedguide/ prostate-cancer-survival-rates>. (Consulta: mayo de 2016).

3. Torres L, Rojas-Martínez R, Escamilla-Nuñez C, De la VaraSalazar E, Lazcano-Ponce E. Tendencias en la mortalidad por cáncer en México de 1980 a 2011. Salud Pública Méx. 2014;56(5).

4. Parker C, Gillessen S, Heidenreich A, Horwich A. Cancer of the prostate: ESMO Clinical Practice Guidelines for diagnosis, treatment and follow-up. An Oncol. 2015;(Suppl 5:69-v77)

5. Islami F, Moreira D, Boffetta P-Freedland S. A Systematic Review and Meta-analysis of Tobacco Use and Prostate Cancer Mortality and Incidence in Prospective Cohort Studies. Eur Urol. 2014;66:1054-1064. 
6. Anderson C, Sternberg I, Karen G, Kim P, Sjoberg D, Vargas $\mathrm{H}$. Age is associated with upgrading at confirmatory biopsy among men with prostate cancer. J Urol 2015:194:16071611.

7. Jiménez MA, Solares M, Martínez $P$, Aguilar J, Martínez J, Hinojosa J, y col. Cáncer de próstata. Cancerología 2011;6:13-18.

8. Consejo de Salubridad General. Guía de Práctica Clínica. Diagnóstico y tratamiento del cáncer de próstata en segundo y tercer nivel de atención. México, 2014.

9. Muralidhar V, Ziehr D, Mahal B, Chen Y, Nezolosky, Viswanathan, et al. Association between older age and increasing Gleason Score. Clin Genitourin Can. 2015;13(6):525-30.

10. D'amico A, Whittington R, Malkowicz B, Schultz D, Blank K, Broderick G, Tomaszewski et al. Biochemical outcome after radical prostatectomy, external beam radiation therapy, or interstitial radiation therapy for clinically localized prostate cancer. JAMA 1998;280(11):969-974.

11. Mohler J, Armstrong A, D'amico A, Davis B, Eastham J, Enke C. Prostate Cancer, NCCN Clinical Practice Guidelines in Oncology. Version 1.2016.

12. Pmerantz M. Active surveillance pathologic and clinical variables associated with outcome. Surg Pathol. 2015;8:581-585.
13. Castillo H, Venegas P, Robles S, Gutiérrez J, Landa M, Xochipiltecatl D. Efectividad de la crioterapia para el cáncer de próstata confinado en el órgano. Estudio comparativo. Rev Mex Urol. 2010;70(1):6-10.

14. Valerio $M$, Ahmed $H$, Emberton M, LawrentshuckN, Lazzeri $\mathrm{M}$, Montironi $\mathrm{R}$, et al. The role of focal therapy in the management of localised prostate cancer: a systematic review. Eur Urol 2014;66:732-752.

15. Hurwitz L, Cullen J, Elsamanoudi S, Kim D, Hudak J, Colston $M$, et al. A prospective cohort study of treatment decisionmaking for prostate cancer following participation in a multidisciplinary clinic. Urol Oncol. 2015;34:17-25.

16. Jones JS, Rewcastle JC, Donnelly BJ. Whole gland primary prostate cryoablation: initial result from the cryo on-line data registry. J Urol 2008;180:554-558.

17. Viramontes-Trejo G. Indicaciones y experiencia en crioablación. An Radiol Mex 2011;3:140-145.

18. Xochipiltecatl-Muñoz J, López-Conde L, CastellanosHernández H, Neave-Sánchez E, García-Villa P, De la CruzGutiérrez $S$, y col. Ablación con criocirugía como manejo en cáncer de próstata. Rev Mex Urol 2011;71:64-67.

19. Crioablación de cáncer. [en línea]. Dirección URL: <http:// www.crioablacion.com/pacientes.html>. (Consulta: mayo de 2016).

\section{AVISO PARA LOS AUTORES}

Revista Mexicana de Urología tiene una nueva plataforma de gestión para envío de artículos: https://www.revisionporpares.com/index.php/RMUrol ahí podrá inscribirse a la base de datos administrada por el sistema Open Journal System (OJS) que ofrece las siguientes ventajas para los autores:

- Subir sus artículos directamente al sistema.

- Conocer, en cualquier momento, el estado de los artículos enviados, es decir, si ya fueron asignados a un revisor, aceptados con o sin cambios, o rechazados.

- Participar en el proceso editorial corrigiendo y modificando sus artículos hasta su aceptación final. 